\title{
All-or-none neural mechanisms underlying face categorisation: evidence from the N170
}

Running title: All-or-none generation of the N170

Haiyang Jin ${ }^{1,2 *}$, William G. Hayward ${ }^{3}$, Paul M. Corballis ${ }^{1}$

1 School of Psychology and Centre for Brain Research, University of Auckland, New Zealand.

2 Department of Psychology, New York University Abu Dhabi, Abu Dhabi

3 Department of Psychology, University of Hong Kong, Hong Kong

* Correspondence concerning this article should be addressed to Haiyang Jin, New York University Abu Dhabi, Saadiyat Island, Abu Dhabi, United Arab Emirates. E-mail: haiyang.jin@outlook.com.

This article has been accepted for publication in Cerebral Cortex Published by Oxford University Press. Its content may be different from the final version. https://doi.org/10.1093/cercor/bhac101.

https://haiyangjin.github.io/en/publication/ 


\begin{abstract}
Categorisation of visual stimuli is an intrinsic aspect of human perception. Whether the cortical mechanisms underlying categorisation operate in an all-or-none or graded fashion remains unclear. In this study, we addressed this issue in the context of the face-specific N170. Specifically, we investigated whether N170 amplitudes grade with the amount of face information available in an image, or a full response is generated whenever a face is perceived. We employed linear mixed-effects modelling to inspect the dependency of N170 amplitudes on stimulus properties and duration, and their relationships to participants' subjective perception. Consistent with previous studies, we found a stronger N170 evoked by faces presented for longer durations regardless of subjective confidence in perceptual categorisation. However, further analysis with equivalence tests revealed that this duration effect was eliminated when only faces perceived with high confidence were considered. Therefore, previous evidence supporting the graded hypothesis is more likely to be an artefact of mixing heterogeneous "all" and "none" trial types in signal averaging. These results support the hypothesis that the N170 is generated in an all-or-none manner, and, by extension, suggest that categorisation of faces may follow a similar pattern.
\end{abstract}

\title{
Keywords
}

equivalence tests; event-related potentials; face categorisation; graded responses; subjective perception 


\section{Introduction}

The ability to categorise environmental stimuli is a fundamental function of human perceptual systems. Perceptual categorisation allows us to organize the rich and dynamic inputs from the environment and supports higher-level cognitive processes such as learning and memory (Retter et al., 2020). A critical question is whether the neural mechanisms subserving categorisation function is in a graded or all-or-none fashion. That is, do these system responses grade with the amount of evidence for some particular category, or do they operate in a more binary fashion, generating a full-strength response only when the evidence for that category passes some threshold? Addressing this question will deepen our understanding of the brain basis for categorisation and advance the construction of corresponding theoretical models.

One canonical neural signature of perceptual categorisation in the visual system is the face-specific enhancement of the N1 component of the event-related brain potential (ERP) typically termed the N170 (e.g., Bentin et al., 1996). The N170 is scalp negativity that is maximal at posterior electrode locations in response to the presentation of a face image. As the nomenclature suggests, the peak amplitude of this component typically occurs around 170 ms poststimulus. It is the earliest reliable ERP correlate of face perception. While the facespecific N170 has been the subject of intense research for nearly three decades (for reviews, see Rossion, 2014; Rossion \& Jacques, 2011; Schweinberger \& Neumann, 2016), it remains uncertain whether this response is generated in a graded fashion, varying in amplitude as a function of the amount or quality of face information available in an image, or is a full response generated whenever a face is perceived.

The idea that the N170 is graded is supported by the observation that lower N170 (or M170, the equivalent response observed in magnetoencephalography) amplitudes are 
recorded when face information is reduced by either reducing the exposure duration (Carbon et al., 2005; Eiserbeck et al., 2021; Tanskanen et al., 2007) or adding noise to the image (Bankó et al., 2011b; Horovitz et al., 2004; Jemel et al., 2003; Navajas et al., 2013; Németh et al., 2014; Philiastides et al., 2006; Philiastides \& Sajda, 2006; Rousselet et al., 2008; Schneider et al., 2007; Tarkiainen et al., 2002). While these reports appear to be consistent with the view that N170 amplitudes are graded with the amount of evidence for a face, on critical evaluation this conclusion may not be warranted. A number of studies of facedetection tasks have reported that, in addition to reducing N170 amplitudes, limiting the amount of information available also results in poorer behavioural performance (Bankó et al., 2011a; Eiserbeck et al., 2021; Nasr, 2010; Retter et al., 2020; Rousselet et al., 2008), and that N170 amplitude also varies positively with behavioural performance (Bankó et al., 2011a; Eiserbeck et al., 2021; Nasr, 2010; Philiastides \& Sajda, 2006; Rousselet et al., 2008; Tanskanen et al., 2007). There are two possible explanations for the relationships between the quality of face information, the amplitude of the face-specific N170, and behavioural performance on face categorisation tasks. At the single-trial level, reducing the quality of face information in the image may elicit a weaker "face" response - and thus a lower-amplitude N170 compared to trials with higher-quality images (i.e., the N170 is a graded response reflecting the strength of the categorisation response) so the average N170 across trials is commensurately lower in amplitude. Alternatively, the relationship between face information and N170 amplitude could be an artefact of the signal averaging inherent in extracting visual evoked potentials. That is, a full-strength N170 may be generated on every trial on which a face in perceived (i.e., the N170 is an all-or-none response reflecting the perception of a face), but since fewer faces are detected on trials with reduced face information, signal averaging results in an N170 that is lower in amplitude for these trials than it is for trials with more face information. While some studies have tried to resolve these possibilities by only 
computing N170 for trials in which a face was correctly perceived (Fisch et al., 2009; Navajas et al., 2013; Rodríguez et al., 2012), it remains possible that the N170 amplitude might still be biased by correct guessing in these studies. To distinguish more completely between these possibilities, it is critical to take both behavioural performance and signal averaging across trials into account.

To disentangle these alternatives, we employed linear-mixed modelling with nullhypothesis significance testing and equivalence tests to examine the dependency of the N170 on face and house images with different durations while participants reported subjective perceptions on each trial. We first inspected this dependence averaged across subjective confidence to replicate previous findings corroborating the graded hypothesis, and then repeated the analysis only for high subjective confidence to exclude contamination from guessing. Moreover, we compared the "full" N170 for different durations in the same blocks to mitigate any effects of experimental context. Overall, our findings support the all-or-none hypothesis for N170 generation.

\section{Materials and Methods}

\subsection{Participants}

We recruited 35 participants for this study, but subsequently excluded seven because their performance failed to meet our inclusion criterion. Specifically, we instructed participants to press Key 1 only when they were sure that the stimulus was a face. For these seven participants, accuracy when pressing Key 1 did not meet our criterion of $95 \%$ correct $(M=76 \%$, range: $49 \%-94 \%)$, so they were not included in further analyses. 
The remaining twenty-eight participants (20 females, age range: $18-32$ years, $M_{\text {age }}=$ 22.32) all had normal or corrected-to-normal vision, participated in the experiment in exchange for course credits or supermarket vouchers, and gave written consent before completing the tasks. This study was approved by the University of Auckland Human Participants Ethics Committee (Reference Number 016833).

\subsection{Stimuli}

The stimuli consisted of 40 photographs of Caucasian (20 female) faces from Ge and colleagues (2009) and 40 house images from Caltech Houses Database (Helle \& Perona, 2000). For face images, we removed all external features, such as ears, hair, and necks by masking the image with an oval window $\left(200 \times 256\right.$ pixels, $\left.4.94^{\circ} \times 6.32^{\circ}\right)$. We applied the same oval window to the house images to ensure that all stimuli shared the same outline. In addition, we converted all the face and house images to greyscale and balanced their luminance using the SHINE toolbox (Willenbockel et al., 2010) in Matlab. We created a scrambled version of each image by randomizing all pixels of each image within the oval window using a custom Matlab script (available: https://osf.io/nxav8/). We show example face and house stimuli and their corresponding scrambled images in Figure 1A. In addition to these stimuli, we created a further four face (two female) and four house images to use in practice trials. We displayed all stimuli against a $7^{\circ} \times 7^{\circ}$ field of partially overlapping circles, which varied in size, location, and shade of grey (Figure 1B). A mask that was identical in composition to this background appeared before and after stimulus presentation.

\subsection{Apparatus}

We administered the experiments using E-prime 2.0 (Version 2.0.8.74, Psychology Software Tools, Pittsburgh, PA). Stimuli were displayed on a Samsung Sync Master HDTV 


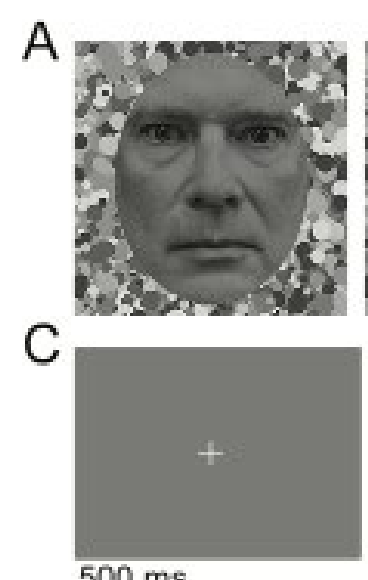

$500 \mathrm{~ms}$
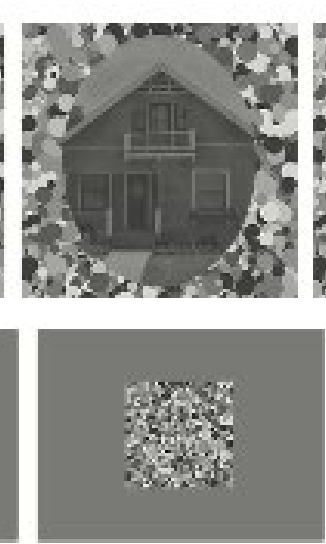

$200-400 \mathrm{~ms}$


$B$
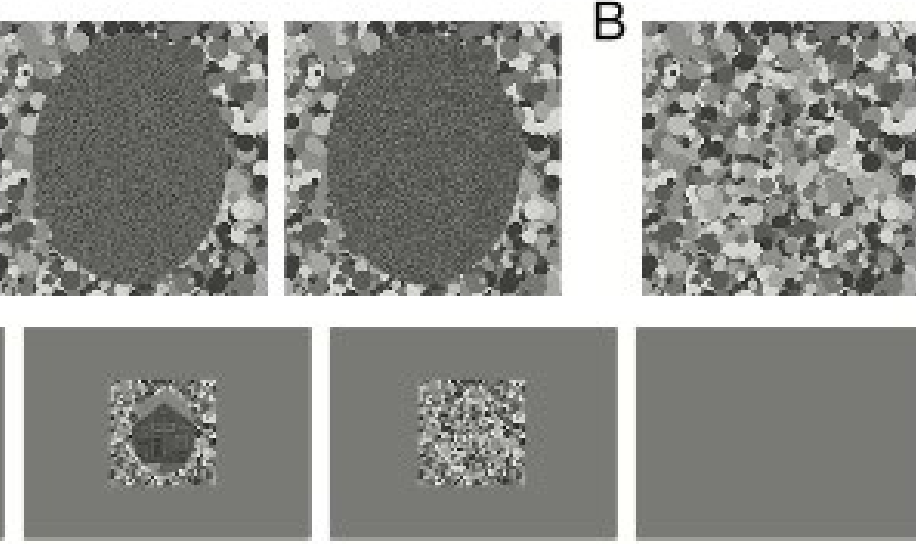

17 or $200 \mathrm{~ms}$



Until Respond

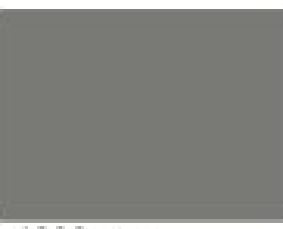

$1000 \mathrm{~ms}$

Figure 1. Stimuli and procedures in this experiment. $A$, Example face and house stimuli, as well as their corresponding scrambled versions, placed on the same masking background. Scrambled stimuli were created by randomizing all pixels within the oval contour. The face example image was not used in the experiment. $B$, The masking image. $C$, The trial procedure in this experiment.

monitor $(\mathrm{P} 2270 \mathrm{HD})$ with $1920 \times 1080$ resolution and a refresh rate of $60 \mathrm{~Hz}$. The background colour of the screen was grey (RGB values: 128, 128, 128). Participants were seated on a comfortable chair in a dimly lit, electrically shielded room (Belling Lee - Model L3000, Enfield, England). Responses were collected via the keyboard. The viewing distance between participants and the screen was maintained at about $57 \mathrm{~cm}$, so that $1 \mathrm{~cm}$ on the screen subtended one degree of visual angle.

Electroencephalography (EEG) was recorded continuously (1000 Hz sample rate; 0.1 $400 \mathrm{~Hz}$ analog bandpass, 400-M $\Omega$ input impedance) throughout each session using 128channel $\mathrm{Ag} / \mathrm{AgCl}$ electrode nets (Tucker, 1993) and amplifiers from Electrical Geodesics Inc. (Eugene, Oregon, USA). Electrode impedances were kept below $40 \mathrm{k} \Omega$, an acceptable level for this system (Ferree et al., 2001). Common vertex (Cz) was used as a reference. 


\subsection{Procedure}

Each trial (Figure 1C) in this study started with a fixation cross displayed for 500ms. This was followed by a pre-stimulus mask with variable duration $(200 \mathrm{~ms}, 250 \mathrm{~ms}, 300 \mathrm{~ms}$, $350 \mathrm{~ms}$, or $400 \mathrm{~ms})$. Then the experimental stimulus was presented superimposed on the background for either $17 \mathrm{~ms}$ or $200 \mathrm{~ms}$, followed by the post-stimulus mask which remained visible until response. We asked participants to judge whether the stimulus was a face or a house by pressing one of five response keys. They were to press Key 1 if they were sure the stimulus was a face, Key 2 if they were not sure, but thought it was a face, Key 5 if they were sure it was a house, Key 4 if they were not sure, but thought it was a house, and Key 3 if they had no idea what it was. The interval between trials was $1000 \mathrm{~ms}$.

We employed a 2 (Type: intact vs. scrambled) $\times 2$ (Category: faces vs. houses $) \times 2$ (Duration: $17 \mathrm{~ms}$ vs. $200 \mathrm{~ms}$ ) within-subject design in this study. There were four blocks in total, and each consisted of 320 trials. In the first two blocks, all stimuli were presented for $17 \mathrm{~ms}$. Every intact stimulus was repeated three times, while every scrambled stimulus was displayed once in each of the first two blocks. In the last two blocks, every stimulus was presented once in each condition. It took about one hour to finish all these trials. In addition, prior to the main experiment, participants completed 32 practice trials, in which the practice stimuli were presented for $17 \mathrm{~ms}$. No feedback was provided at the single-trial level, and the responses were summarized at the end of the practice.

\subsection{EEG data pre-processing}

Pre-processing of EEG data was conducted using EEGLAB (Version 14.1.2, Delorme \& Makeig, 2004) in Matlab (Version 9.3, MathWorks, Natick, MA). All the steps, except for rejecting the independent components based on the results of independent component 
analysis (ICA), were completed using Matlab scripts (available: https://osf.io/fvtc3/) in the High-Performance Computing cluster of New Zealand eScience Infrastructure.

The pre-processing pipeline mainly followed "Makoto's suggestion" from (Miyakoshi, 2019). First, the options of EEGLAB were changed to using double precision. After loading the raw EEG data, the timestamps of all triggers were shifted 50ms earlier to correct for amplifier and display delays. The amplifier delay was due to the amplifier taking time to process signals, which is reported by EGI to be $36 \mathrm{~ms}$ for a $1000 \mathrm{~Hz}$ sampling rate. The display delay was caused by the monitor taking time to display the stimuli. This delay time was measured by comparing the differences between the time point at which triggers of a stimulus were sent by E-prime and the time at which a photocell detected the change in luminance on the monitor, which was $14 \mathrm{~ms}$ on average. In total, the delay time is $50 \mathrm{~ms}$. Then the EEG data were downsampled from $1000 \mathrm{~Hz}$ to $250 \mathrm{~Hz}$ and bandpass filtered (1-50 Hz). Next, the channel location information was loaded, and the line-noise frequencies at $50 \mathrm{~Hz}$, $100 \mathrm{~Hz}, 150 \mathrm{~Hz}, 200 \mathrm{~Hz}$, and $250 \mathrm{~Hz}$ were removed with the CleanLine (Version 1.04) plugin. Bad channels were identified and removed with the clean_rawdata (Version 1.00) plugin, and then interpolated. Next, the EEG data were re-referenced to the average of all the channels. ICA was conducted on the continuous EEG data with AMICA (Version 1.5.1), and the results saved for further use. Note that a high-pass filter of $1 \mathrm{~Hz}$ was utilized in the initial part of the pre-processing analysis because high-pass filtered data at $1-2 \mathrm{~Hz}$ works better for ICA (Winkler et al., 2015). However, previous research also found that high-pass filtering (above $0.1 \mathrm{~Hz}$ ) can distort the early ERP components (Acunzo et al., 2012). Therefore, we repeated all the above pre-processing steps except for the ICA analysis on the original data, but with a high-pass filter frequency of $0.1 \mathrm{~Hz}$ instead of $1 \mathrm{~Hz}$. Then the ICA results from the initial pass (filtered with high-pass at $1 \mathrm{~Hz}$ ) were applied to the data filtered with high-pass at $0.1 \mathrm{~Hz}$, as recommended by Makoto (Miyakoshi, 2019). The equivalent-dipole source 
localization of independent components was calculated with dipfit (Version 3.0) plugin, which was followed by identifying the components representing bilateral source activities with fitTwoDipoles (Version 0.01) plugin (Piazza et al., 2016). We used the dipole information to reject components that did not appear to have a brain source. Next, the continuous EEG data were segmented into $1500 \mathrm{~ms}$ epochs, starting $500 \mathrm{~ms}$ before the stimulus onsets. After completing all the above steps, we transferred the data to a local computer for further processing. Independent components identified as artifacts, such as eye

blinks, horizontal eye movements, heartbeats, muscle contractions, and so on, were identified with SASICA (Chaumon et al., 2015), which implemented its own selection algorithms, and other two automated methods: ADJUST (Mognon et al., 2011) and FASTER (Nolan et al., 2010). Manually rejecting artefactual effects was mainly based on the results of SASICA and guided by (Pion-Tonachini et al., 2019). After removing the artifact components, the data were reloaded to the cluster for further pre-processing. Improbable data were identified by joint probability and marked for all the channels. A linear detrend was applied from $200 \mathrm{~ms}$ prior to $996 \mathrm{~ms}$ after stimulus onset for each epoch. As the last step of pre-processing, the average voltage during the $200 \mathrm{~ms}$ prestimulus baseline was subtracted from each data point in each epoch.

\subsection{Time windows, peak channels, and mean amplitudes}

We used a data-driven method to identify appropriate time-windows for the N170 and P1 components that are unbiased by condition. To identify the N170 time window, we chose P7 (E58) and P8 (E96) as the likely peak channels based on previous literature (Jemel et al., 2003; Nemrodov et al., 2016; Rossion \& Jacques, 2008). The cluster channels for P7 (E58) included electrodes E58, E65, E59, E51, E50, E57, and E64. The cluster channels for P8 (E96) included electrodes E96, E101, E97, E91, E96, E95, and E100. A grand-average 
waveform for the cluster of channels centred on P7 and P8 was obtained by averaging the data for the entire epoch across participants, conditions, and cluster channels. Then we identified the minimum (i.e., most negative) amplitude of this grand-average waveform between $100 \mathrm{~ms}$ and $250 \mathrm{~ms}$ poststimulus. To compute the amplitude of the N170, we initially defined an integration window based on the full-width half-maximum of the component. If the width of this window was outside the range of $36-40 \mathrm{~ms}$ (a reasonable window for N170), the height-width ratio was adjusted iteratively until the integration window was between $36 \mathrm{~ms}$ and $40 \mathrm{~ms}$ wide.

After setting the N170 integration window, we created a grand mean scalp distribution for this period across all participants and conditions to allow visual confirmation of the peak channels. We observed that the centres of the negative peak activity were around PO7 (E65) and PO8 (E90), rather than our initial assumption of P7 and P8. Therefore, we updated the channel clusters to centre on PO7 (channels E65, E70, E66, E59, E58, E64, and E69), and PO8 (E90, E96, E91, E84, E83, E89, and E95). We then re-checked the N170 integration window using the same procedure outlined above using the updated peak channels. As a result, the integration window for the N170 was defined as $152-192 \mathrm{~ms}$ (time window size: $40 \mathrm{~ms})$ poststimulus.

We used an analogous procedure to define the integration window for the P1 component, which we include in the analysis as a control, since we do not expect P1 to be modulated by perceptual categorisation. We initially chose PO7 (E65) and PO8 (E90) as the likely scalp maxima of P1 based on previous literature (Bankó et al., 2011a; Kuefner et al., 2010; Rossion \& Caharel, 2011; Smith, 2012) and the maximum amplitude value of the grand averaged epoch was identified between $70 \mathrm{~ms}$ and $140 \mathrm{~ms}$ after stimulus onsets. As a result, we set the integration window for P1 as $96 \mathrm{~ms}-136 \mathrm{~ms}$ (time window size: $40 \mathrm{~ms}$ ) poststimulus. The grand-average scalp distribution for this window confirmed that the peaks 
of P1 activity were at the PO7 and PO8 electrodes. Accordingly, the channel clusters for the P1 were identical to those identified for N170.

We defined the amplitudes for the two ERP components (P1 and N170) as the mean amplitudes within their respective integration windows. We measured the amplitudes in each window for every trial, and then submitted the single-trial mean amplitudes to linear mixed modelling (LMM) for further analysis.

\subsection{Statistical analyses}

\subsubsection{Linear mixed modelling}

We conducted linear mixed modelling (LMM), instead of repeated-measure analyses of variance (rm-ANOVA), to assess single-trial mean amplitudes. There are several advantages of LMM which make this technique popular as an alternative to rm-ANOVA (Aarts et al., 2014; Baayen et al., 2002; Boisgontier \& Cheval, 2016; Jaeger, 2008; Kristensen \& Hansen, 2004; Quené \& van den Bergh, 2004). The specific benefit of applying LMM in this study is that, compared with rm-ANOVA, LMM can yield unbiased results in the presence of missing data without the need to remove participants (Brauer \& Curtin, 2018; Kliegl et al., 2011; Linck \& Cunnings, 2015; Magezi, 2015; Matuschek et al., 2017). In this study, we expected that when intact face stimuli were presented for $17 \mathrm{~ms}$, some participants may never perceive the stimuli with high subjective confidence, and thus not use the Key 1 or Key 5 responses at all. We also thought it possible that some ratings may not be used by all participants. Another advantage of using LMM is that they can model amplitudes at the level of single observations and estimate multiple random effects. Estimating both participant and stimulus random effects maximizes data use and allows generalization of fixed effects to other similar participants and stimuli. 
The general procedures to obtain an optimal model for each dependent variable follow the recommendations of Bates, Kliegl, and colleagues (2015) and Matuschek and colleagues (2017). First, the maximal model is created, in which the random effect structure includes all by-participant and by-item random intercepts and random slopes. If the maximal model fails to converge, the corresponding zero-correlation-parameter (ZCP) model, in which the correlations between random effects are forced to be zero, is fitted. Then the random effects that do not significantly explain the data are removed from the ZCP model, resulting in the reduced model. The extended model is then built by adding back the correlations between the remaining random effects in the reduced model. If the extended model still cannot converge, the random effects with the smallest amount of variance are removed one-by-one until a converged model is achieved, resulting in the final extended model. Next, the reduced and extended models are compared, and the one that explains data better (with smaller Akaike's Information Criterion; Akaike, 1998) is defined as the optimal model. Follow-up comparisons are conducted based on this optimal model.

In this study, we are interested in both the overall ERPs and in the ERP differences between faces and nonface objects. First, to inspect how P1 and N170 amplitudes varied when intact faces were presented for different durations and distinct subjective confidence, we performed pairwise contrasts for all Duration and Confidence levels separately on the results of the optimal LMM for intact faces. Second, to test the dependency of the aspect of the ERP that could be specifically attributed to face processing (here defined as the difference between the face-related and house-related P1 and N170 amplitudes) for intact stimuli on durations and subjective confidence, we computed "pairwise interaction contrasts" between Category and Duration (or Confidence) for intact faces (i.e., simple interaction analysis; Jin, 2021). For instance, the pairwise interaction contrast between Category and Duration in this 
study is equivalent to ( $17 \mathrm{~ms}$ faces $-17 \mathrm{~ms}$ houses $)$ - (200ms faces $-200 \mathrm{~ms}$ houses $)$, i.e., the differences of face-minus-house ERP component between durations.

\subsubsection{Null-hypothesis significance tests and equivalence tests}

Null hypothesis significance testing (NHST) remains one of the most popular inferential statistical methods used by researchers. When the probability (i.e., p-value) of observing the data and more extreme under the null hypothesis is small (less than some predefined $\alpha$ level), we reject the null hypothesis and infer that the alternative hypothesis is true. However, when that probability is not small enough, we cannot conclude whether the null hypothesis is true or whether there is insufficient evidence to make any decision (Cohen, 1994).

In this study, we do expect that some null effects. For example, comparable N170 amplitudes may be generated for faces presented for different durations when they are perceived with high subjective confidence. Such effects can never be positively supported by conventional NHST. Therefore, in addition to conventional NHST, we employ equivalence tests (ET) in this study to test whether the effect is equivalent to a region of null effects (Lakens, 2017; Lakens et al., 2018; Mara \& Cribbie, 2012; Meyners, 2012; Rogers et al., 1993; Seaman \& Serlin, 1998; Weber \& Popova, 2012). ET essentially performs two onesided tests to determine whether the observed effects are outside a region that is believed to be equivalent to a null effect. In other words, the null hypothesis being tested in ET is that the observed effect is smaller than the lower boundary of the equivalence region or larger than its upper boundary. In this study, we define this region of practical equivalence to zero as [$0.5 \mu \mathrm{V}, 0.5 \mu \mathrm{V}]$, which, we think, is reasonably small considering that ERP data are usually noisy. If the probability that the observed effects being outside the region is low (i.e., $p$ values for the two one-sided tests are both smaller than the conventional $\alpha$ level of 0.05 ), we 


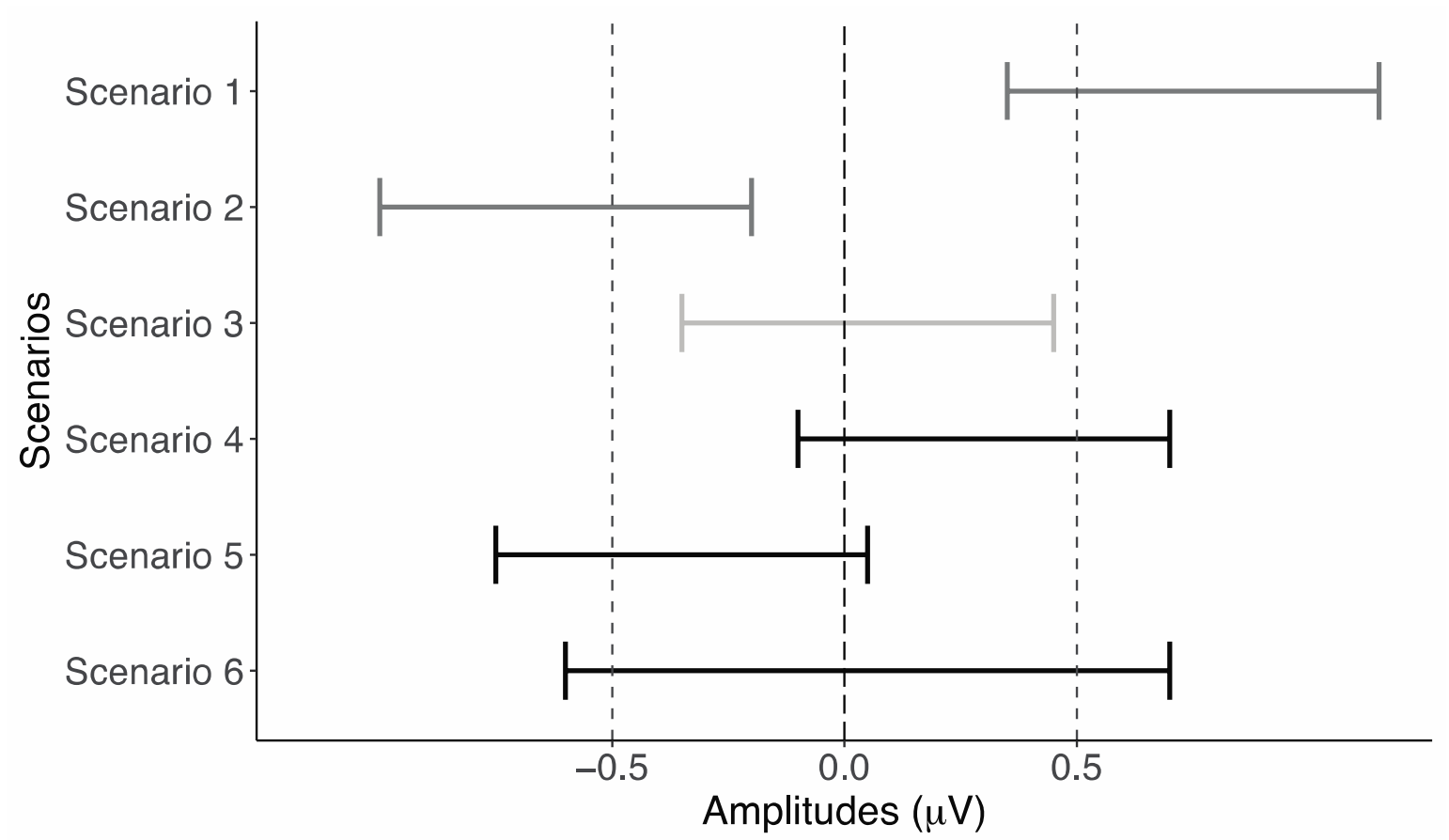

Figure 2. Scenarios of results obtained from conventional null hypothesis significance testing (NHST) and equivalence tests (ET). The black long-dashed vertical line denotes the effect of zero, which represents the null hypothesis in NHST. The grey dashed vertical lines denote the lower and upper boundaries $[-0.5,0.5]$ of the region equivalent to the null effect in equivalence tests. The horizontal error bars denote the confidence intervals of different effects. Scenario 1 and 2 denote that the NHST is significant but the ET is not, which provides the evidence that the effect of interest is different from 0. Scenario 3 denotes that the ET is significant but the NHST is not, which provides the evidence that the effect of interest is equivalent to the null effect. Scenario 4-6 denote that the effects are inconclusive with the evidence from NHST and ET.

will reject that hypothesis and infer that the observed effect is statistically equivalent to the null effect. If the probability of any direction is not small enough, we cannot infer the effect is equivalent to the null effect.

In summary, we employ both conventional NHST and ET in this study to examine whether an effect is significantly different from zero and whether it is practically equivalent to the region of null effects, respectively. There are (at least) six possible scenarios that can be obtained by combining conventional NHST and ET (see Figure 2 for a schematic 
representation of these possibilities). Scenarios 1 and 2 reflect instances in which the null hypothesis is rejected. In these instances, we only report the results of NHST. Scenario 3 reflects cases in which there is evidence in favour of the null effects. Here, we only report the results of ET. Since two one-sided tests are performed in ET, we only report the results of the one-sided test whose absolute $t$-value is smaller and thus $p$-value is larger (Lakens et al., 2018). For the other scenarios, in which the null hypothesis can neither be rejected nor supported, and, therefore, the effects are inconclusive, we report results of both NHST and ET.

\subsubsection{Software}

We performed all statistical analyses using R (R Core Team, 2018) and RStudio (RStudio Team, 2019). Data were tidied up with the Tidyverse package (Wickham, 2017). We performed LMM using the lme4 package (Bates, Mächler, et al., 2015) and follow-up comparisons and ET with the emmeans package (Lenth, 2019). The R Markdown output file is available at https://osf.io/zv6kg/.

\section{Results}

\subsection{Allocation of keypress responses}

The allocation of responses in each condition is summarized in Figure 3A. Inspection of this figure reveals that, for brief $(17 \mathrm{~ms})$ presentations, faces were perceived more frequently and with higher confidence than houses. Specifically, when intact faces were presented, participants reported being sure that they had seen a face (Key 1) on about half of trials $(M=0.469,95 \%$ confidence interval $(C I):[0.346,0.591])$, and unsure, but thought the 

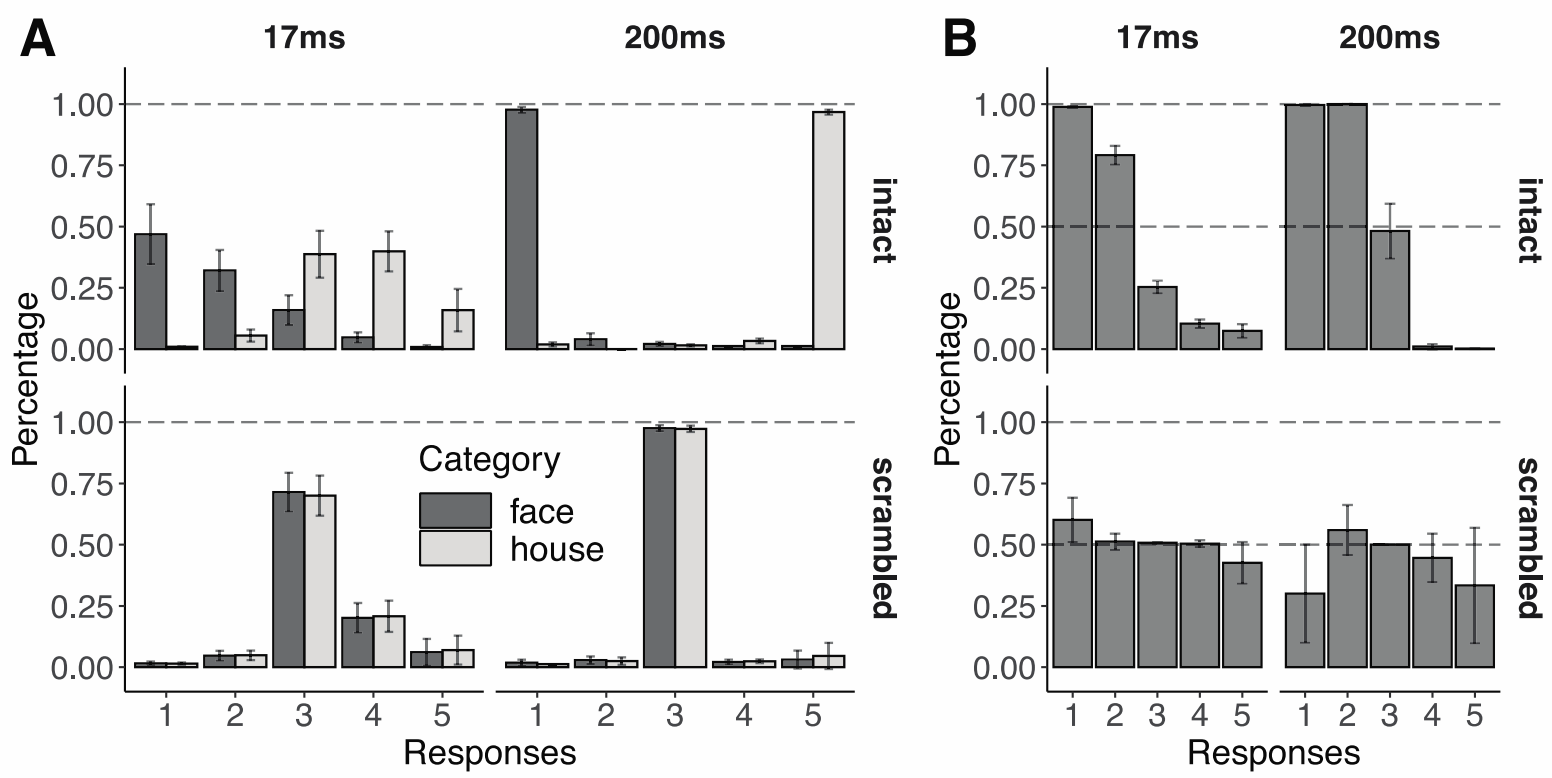

Figure 3. Allocation of keypress responses. Response 1 denotes participants were sure the stimulus was a face, response 2 denotes participants were not sure but thought it was a face, response 3 denotes participants had no idea what it was, response 4 denotes participants were not sure but thought it was a house, and response 5 denotes participants were sure it was a house. Error bars represent $95 \%$ confidence intervals. Panel $\boldsymbol{A}$ displays the averaged responses within each condition. Panel $B$ depicts the percentage for each response and condition of trials in which the stimulus was a face. For instance, the leftmost column in the first row of Panel $B$ shows that almost all intact stimuli were faces when participants responded with Key 1.

stimulus was a face (key 2$)$ on a further third of the trials $(M=0.321,95 \% C I:[0.236$, 0.405]). When intact houses were presented, participants were less confident. The most common responses were that they were unsure, but thought the stimulus was a house (Key 4; $M=0.399,95 \% C I:[0.317,0.481])$ or had no idea what were presented (Key $3 ; M=0.387$, 95\% CI: $[0.292,0.483])$. When the corresponding scrambled images were presented for $17 \mathrm{~ms}$, participants most commonly responded that they had no idea what stimulus was presented (Key 3; scrambled faces: $M=0.714,95 \% C I:[0.635,0.793]$; scrambled houses: $M$ $=0.700,95 \% C I:[0.618,0.782])$. As expected, when the stimuli were presented for $200 \mathrm{~ms}$, participants were essentially perfect in discriminating intact faces from intact houses, and had high confidence in their responses (Key 1; faces: $M=0.977,95 \% C I$ : $[0.965,0.989]$; houses: 
$M=0.967,95 \% C I:[0.957,0.977])$. When the images were scrambled, the participants once again consistently reported having no idea whether they depicted faces or houses (Key 3; faces: $M=0.975,95 \% C I$ : [0.963, 0.988]; houses: $M=0.973,95 \% C I:[0.960,0.986])$.

Figure 3B depicts the percentage of stimuli that were faces for each response in the intact and scrambled conditions. For instance, the leftmost column in the first row depicts the percentage of stimuli being intact faces when participants responded with Key 1 . If participants were following instructions appropriately, they should have pressed Keys 1 and 5 only when they were completely sure of the stimulus. Note that we removed participants who failed to reach $95 \%$ accuracy for these responses before conducting any further analysis (see Participants above). Indeed, regardless of stimulus duration, when participants responded with Key 1, almost all the stimuli were intact faces $(17 \mathrm{~ms}: M=0.988,95 \% C I=[0.983$, 0.994]; 200ms: $M=0.996,95 \% C I=[0.993,0.999])$, while only very few stimuli were faces when participants responded with Key 5 (17ms: $M=0.074,95 \% C I=[0.020,0.128] ; 200 \mathrm{~ms}$ : $M=0.002,95 \% C I=[0.000,0.004])$. In addition, we also instructed participants to select Key 3 when they were unable to categorize the stimuli. For scrambled stimuli, this resulted in chance performance for Key 3 responses (17ms: $M=0.508,95 \% C I=[0.500,0.515] ; 200 \mathrm{~ms}$ : $M=0.501,95 \% C I=[0.499,0.502])$.

These data confirm that participants were using the subjective-confidence scale appropriately and distributing their responses across the five response options. Critically, the variability in subjective confidence when the stimuli were presented for $17 \mathrm{~ms}$ enables us to explore further the associations between subjective confidence and ERP amplitudes. 


\subsection{ERP amplitudes averaged across responses}

We used LMM to analyse the amplitudes of the P1 and N170 components of the ERP in three different ways. First, we collapsed across response options to replicate the effects of stimulus duration reported in previous literature (Carbon et al., 2005; Mitsudo et al., 2011; Tanskanen et al., 2007), in which N170 amplitudes were larger for longer durations compared with shorter durations. While our main focus was on modulation of N170, we also analysed P1 amplitudes (which we do not expect to be modulated by face stimuli; Rossion \& Caharel, 2011) to account for any effects that result from general amplification of the ERP as a function of stimulus duration. The grand average ERP waveforms and topographic maps corresponding to the P1 and N170 time-windows are shown in Figure 4D and 4C, respectively. We fitted amplitudes for each component using LMM and the results were submitted to pairwise contrasts and pairwise interaction contrasts to test the influence of stimulus properties on P1 and N170 amplitudes, as well as on face-minus-house ERP component.

\subsubsection{P1 amplitudes}

The estimated marginal means of P1 amplitude for each condition are depicted in Figure 4A. To examine the dependency of P1 amplitudes on stimulus properties, we performed pairwise contrasts corrected by the Bonferroni methods for two tests. Results revealed that the differences in $\mathrm{P} 1$ amplitudes evoked by intact faces between $17 \mathrm{~ms}$ and $200 \mathrm{~ms}$ conditions were statistically equivalent to the null effect for the left-hemisphere electrodes $(t(123.0)=2.61, p=.01, b=-0.07,90 \% C I=[-0.40,0.25])$, but remained inconclusive for the right-hemisphere electrodes (NHST: $t(123.0)=-1.24, p=0.44$; ET: $t(123.0)=1.80, p=.07, b=-0.20,90 \% C I=[-0.53,0.12])$. Moreover, to examine the dependency of P1 amplitude differences between faces and houses on stimulus properties, we 


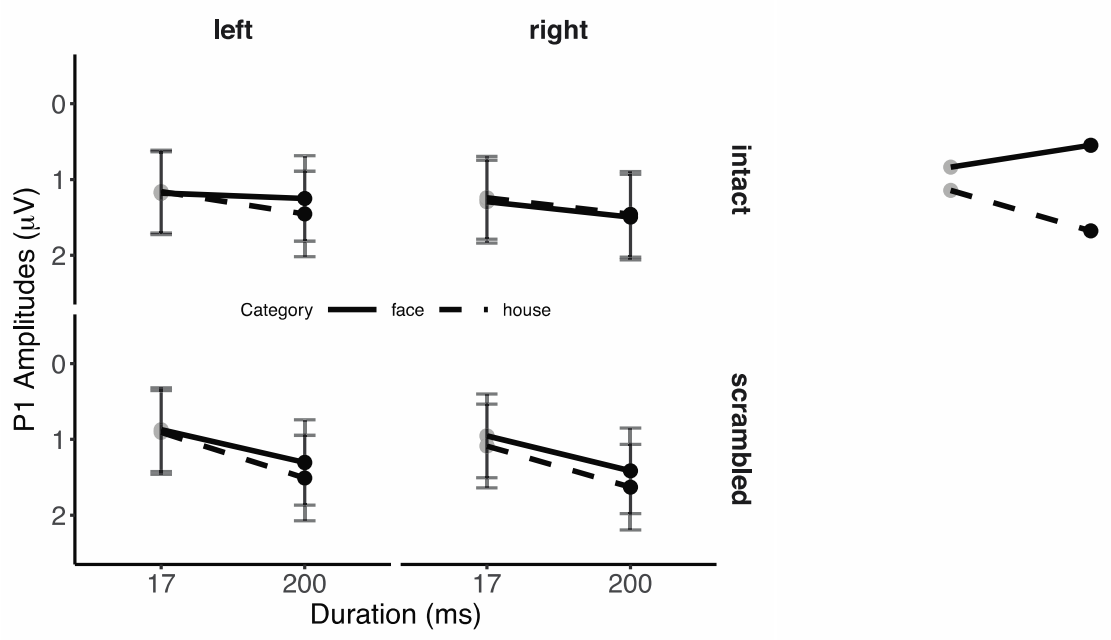

ambled

$17 \mathrm{~ms} \quad 200 \mathrm{~ms}$

Figure 4. ERP results as a function of Hemisphere (left vs. right), Type (intact vs. scrambled), Category (faces vs. houses), and Duration (17ms vs. 200ms) across behaviour responses. $A$ and $B$ depict the estimated marginal means of $\mathrm{P} 1$ and $\mathrm{N170}$ amplitudes, respectively; error bars represent $95 \%$ confidence intervals. $C$, Topographic maps corresponding to the P1 (left panel) and N170 (right panel) components. The grey dots in the schematic brain denote the channel locations for both P1 and N170 on both hemispheres. $D$, The grand average ERP waveforms. The grey dashed rectangular denotes the timewindow for the P1 (96ms-136ms), while the grey solid rectangular denotes the time windows for the N170 (152-192ms). Please see Figure S1 in supplementary materials for colour topographic maps. 
performed pairwise interaction contrasts between Category and Duration for intact stimuli, also corrected by the Bonferroni method for two tests. No significant face-related modulation of the P1 component by stimulus duration was found for the right-hemisphere electrodes $(t(266.2)=-3.12, p=.002, b=0.01,90 \% C I=[-0.29,0.32])$, whereas this modulation was inconclusive for the left-hemisphere electrodes (NHST: $t(266.2)=1.44, p=0.30$; ET: $t(266.2)=-1.76, p=.08, b=0.22,90 \% C I=[-0.08,0.53])$. Both analyses suggest that, neither the overall P1 nor the face-minus-house P1 components were affected by durations, implying that any differences found for N170 amplitudes later could not be attributed to generalised amplification of the ERP for longer stimulus duration.

\subsubsection{N170 amplitudes}

The estimated marginal means of N170 amplitudes in each condition are displayed in Figure 4B. In general, longer stimulus duration was associated with a weaker (lower amplitude) N170, except when the stimuli were intact faces. Pairwise contrasts, corrected using the Bonferroni method for two tests, confirm that the N170 amplitudes evoked by intact faces were weaker for $17 \mathrm{~ms}$ relative to $200 \mathrm{~ms}$ presentations for both hemisphere electrodes $($ left: $t(100.1)=2.62, p=.02, b=0.68,95 \% C I=[0.09,1.26]$; right: $t(100.1)=5.02, p<$ $.001, b=1.30,95 \% C I=[0.71,1.88])$. This dependence of N170 amplitude on Duration seems to support the graded hypothesis for N170 generation: longer exposure to faces results in larger N170 amplitude, whereas in all other conditions, longer exposure results in lower amplitude.

Next, we compared the face-minus-house N170 components for different durations via pairwise interaction contrasts between Category and Duration for intact stimuli. These contrasts revealed significant interactions between Category and Duration for both hemisphere electrodes (left: $t(104.1)=8.93, p<.001, b=1.92,95 \% C I=[1.43,2.41]$; right: 
$t(104.1)=10.40, p<.001, b=2.24,95 \% C I=[1.75,2.73])$, suggesting that $\mathrm{N} 170$ amplitudes differences between faces and houses were stronger for $200 \mathrm{~ms}$ than $17 \mathrm{~ms}$ conditions. This result also seems to confirm that the face-minus-house N170 component is graded.

\subsection{The influence of subjective confidence on ERP}

\section{amplitudes}

The previous analyses compared P1 and N170 amplitudes for face and house images - as well as scrambled versions of both - regardless of the participants' responses. While the results are consistent with the idea that the N170 (but not the P1) is graded in amplitude as a function of the amount of face information, it is possible that the N170 is contaminated by including trials in which no face was perceived. This would be expected to reduce the N170 for the short-duration $(17 \mathrm{~ms})$ condition more than the long-duration (200ms) condition, perhaps creating an illusion of a graded response. Accordingly, we repeated the analysis taking subjective confidence into account. As shown in Figure 3A, we mostly observed variability in response when intact stimuli were presented for $17 \mathrm{~ms}$. In particular, most responses for $17 \mathrm{~ms}$ intact faces were Key 1, Key 2, and Key 3, whereas most responses for $17 \mathrm{~ms}$ intact houses were Key 5, Key 4, and Key 3. These keys corresponded to high confidence, low confidence, and guessing for face and house stimuli, respectively. Thus, we converted these responses into a new fixed factor, Confidence, which had three levels: high (Key 1 for faces or Key 5 for houses), low (Key 2 for faces or Key 4 for houses), and guess (Key 3 for both stimuli). Since we also wished to include the $200 \mathrm{~ms}$ intact stimuli conditions as the baseline level, and most responses in these conditions were Key 1 and Key 5 (i.e., high confidence for faces and houses respectively), we further integrated Confidence with Duration to create a new factor, Duration Confidence, with four levels: 17ms_guess, 17ms_low, 17ms_high, and 200ms_high. We did not include scrambled trials in the analyses. 

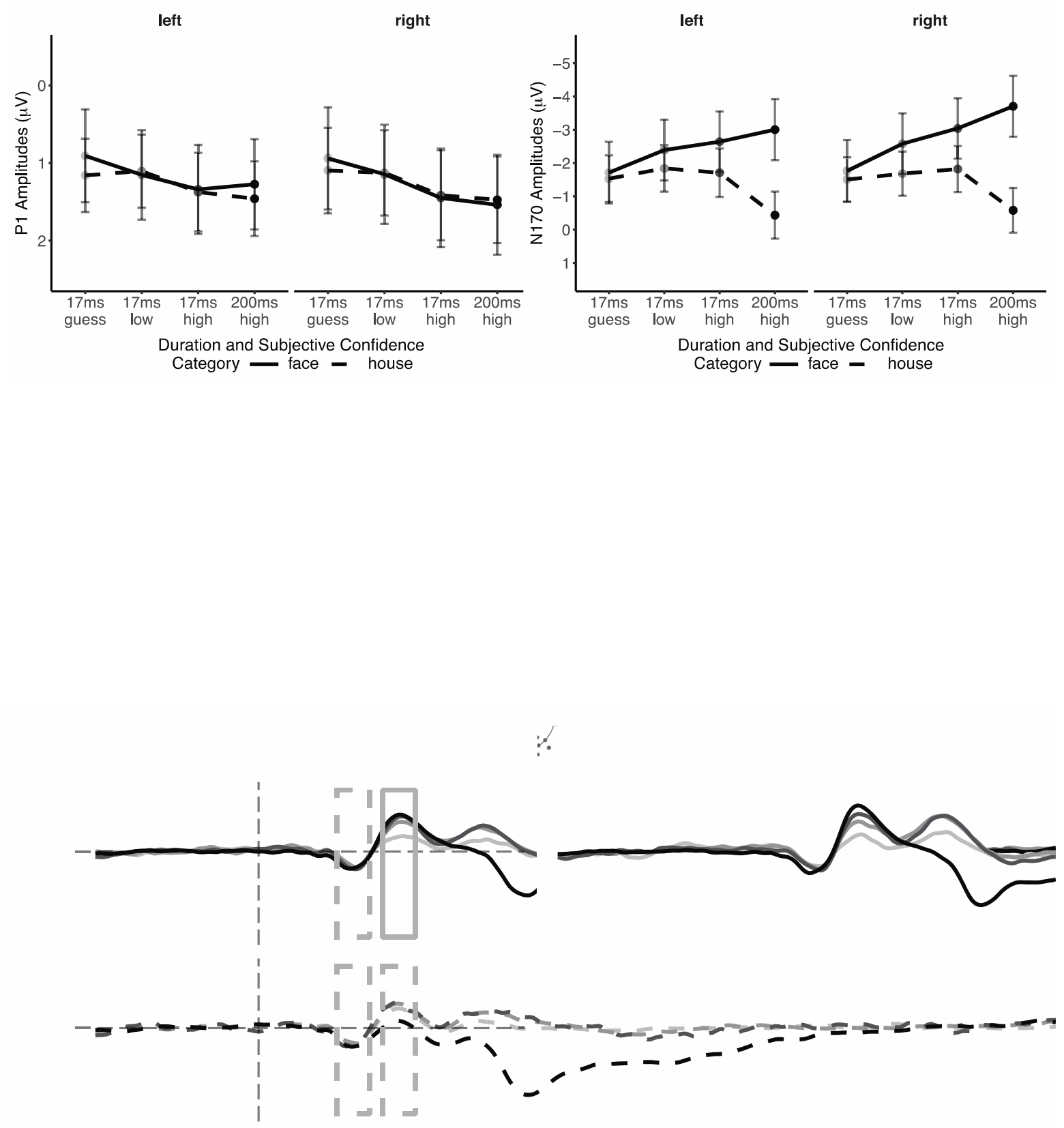

Figure 5. ERP results for intact stimuli as a function of Hemisphere (left vs. right), Category (faces vs. houses), and Duration Confidence (17ms_guess, 17ms_low, 17ms_high, vs. 200ms_high). $A$ and $B$ depict the estimated marginal means of P1 and N170 amplitudes, respectively; error bars represent $95 \%$ confidence intervals. $C$, Topographic maps corresponding to the $P 1$ and $N 170$ components. The grey dots in the schematic brain denote the channel locations for both P1 and N170 on both hemispheres. $D$, The grand average ERP waveforms. The grey dashed rectangular denotes the time-window for the P1 (96ms 136ms), while the grey solid rectangular denotes the time windows for the N170 (152-192ms). Please see Figure S2 in supplementary materials for colour topographic maps. 
The grand average ERP waveforms and topographic maps corresponding to the P1 and N170 time-windows are shown in Figure 5C (topographies) and 5D (waveforms). We analysed the LMM results using pairwise contrasts and pairwise interaction contrasts to test the influence of Duration Confidence on overall ERP amplitudes and the face-minus-house ERP component.

\subsubsection{P1 amplitudes}

The estimated marginal means of P1 amplitude for each condition are depicted in Figure 5A. Similar to the earlier analyses, we performed pairwise contrasts to test the dependency of P1 amplitudes on Duration Confidence, corrected with Bonferroni methods for six tests, and the detailed statistical results are available in Table S1. For the lefthemisphere electrodes, P1 amplitudes in $17 \mathrm{~ms}$ guess conditions were weaker than those in $17 \mathrm{~ms}$ high conditions $(t(20687.5)=-3.14, p=.01, b=-0.43,95 \% C I=[-0.80,-0.07])$.

Differences between 17 low, 17 high and 200_high were statistically equivalent to the null effect $(|t|>2.86, p<.02)$ while the comparisons between $17 \mathrm{~ms}$ guess and $17 \mathrm{~ms} \_$low, as well as between 17ms_guess and 200ms_high, were not conclusive (NHST: $|t|<2.57, p>.06$; ET: $|t|<1.89, p>.18)$. For the right-hemisphere electrodes, P1 differences between 17ms_high and 200_high were statistically equivalent to the null effect $(t(34348.2)=3.76, p<.001, b=-$ $0.09,90 \% C I=[-0.35,0.17])$. Moreover, $\mathrm{P} 1$ amplitudes for $17 \mathrm{~ms}$ guess and $17 \mathrm{~ms}$ low were weaker than those for 17ms_high and 200ms_high $(|t|>2.79, p<.04)$. And P1 differences between 17ms_guess and 17ms_low were not conclusive (NHST: $t(35346.6)=-1.51, p=.79$; ET: $t(35346.6)=2.19, p=.09)$. These results indicate that, in general, weaker P1 amplitudes were associated with lower subjective confidence but the effects were quite small, usually smaller than $1 \mu \mathrm{V}$. 
We also assessed face-minus-house $\mathrm{P} 1$ amplitudes with pairwise interaction contrasts between Category and Duration Confidence, which were corrected with Bonferroni methods for six tests and the detailed statistical results are available in Table S1. For the lefthemisphere electrodes, we found equivalence to null effects only for the comparisons between 17ms_guess and 200ms_high $(t(31977.1)=2.42, p=.05, b=-0.06,90 \% C I=[-$ $0.50,0.37])$ and between $17 \mathrm{~ms} \_$low and $17 \mathrm{~ms}$ _high $(t(7492.4)=-2.43, p=.05, b=0.08$, $90 \% C I=[-0.33,0.49])$; the other comparisons remained inconclusive (NHST: $|t|<1.79, p>$

.44 ; ET: $|t|<1.92, p>.16$ ). For the right-hemisphere electrodes, we found that the differences in face-minus-house P1 amplitudes between the 17ms_low, 17ms_high, and 200ms_high were statistically comparable $(|t|>2.58, p<.03)$, while other comparisons were inconclusive (NHST: $|t|<1.23, p>.99$; ET: $|t|<1.94, p>.15$ ). In comparison to the overall P1 amplitude results, the face-minus-house P1 component was less likely to be influenced by subjective confidence. That is, variations in P1 amplitude as a function of subjective confidence cannot be attributed to modulation of a face-specific response, but are instead likely to reflect general visual processing.

\subsubsection{N170 amplitudes}

The estimated marginal means of N170 amplitudes in each condition are displayed in Figure 5B. We conducted pairwise contrasts to compare the differences in N170 amplitudes evoked by intact faces between levels of Duration Confidence, with Bonferroni corrections for six tests, and the statistical results are available in Table S1. For the left-hemisphere electrodes, N170 amplitudes for 17ms_guess were weaker than all other levels of Duration Confidence $(|t|>4.84, p<.001)$. Differences of N170 amplitudes between 17ms_low and 17ms_high conditions were not conclusive (NHST: $t(27206.8)=2.15, p=0.19$; ET: $t(27206.8)=-2.12, p=0.10)$ and the amplitudes elicited in both of these conditions were 
weaker than those in the 200ms_high condition $(|t|>3.20, p<.009)$. A similar pattern occurred for the right-hemisphere electrodes, with all differences reaching significance. Specifically, N170 amplitudes for 17ms_guess were weaker than all other three levels $(|t|>$ 5.85, $p<.001) . \mathrm{N} 170$ amplitudes for $17 \mathrm{~ms} \_$low were weaker than $17 \mathrm{~ms}$ high and 200ms_high $(|t|>3.95, p<.001)$. Critically, when the subjective confidence was high, N170 amplitudes for the $17 \mathrm{~ms}$ conditions were still weaker than for $200 \mathrm{~ms}(t(36540.1)=5.88, p<$ $.001, b=0.66,95 \% C I=[0.37,0.96])$. These results revealed that stronger (absolute) N170 amplitudes were associated with higher subjective confidence. Notably, even when perceived with similarly high subjective confidence, faces presented for $200 \mathrm{~ms}$ still evoked stronger N170 amplitude compared to $17 \mathrm{~ms}$. This result, too, appears to support the graded hypothesis.

We examined the face-minus-house N170 component using pairwise interaction contrasts between levels of Category for each level of Duration Confidence, corrected using the Bonferroni method for six tests, and the statistical results are available in Table S1. For the left-hemisphere electrodes, face-minus-house component in the 200ms_high condition was stronger than all other three levels $(|t|>8.52, p<.001)$. N170 amplitude in the $17 \mathrm{~ms}$ high condition was also stronger than in $17 \mathrm{~ms}$ _guess condition $(t(8673.1)=-3.52, p=$ $.003, b=-0.75,95 \% C I=[-1.31,-0.19])$. Differences between other levels were inconclusive (NHST: $|t|<2.10, p>.21$; ET: $|t|<0.76, p>.99$ ). For right-hemisphere electrodes, the faceminus-house components differed between all levels except for the comparison between 17ms_low and 17ms_high, which remained inconclusive (NHST: $t(5675.1)=-1.75, p=.49$; ET: $t(5675.1)=-0.96, p>.99)$. Specifically, the N170 was stronger in the 200ms_high condition than the other three levels $(|t|>10.01, p<.001)$. The N170 amplitudes in the $17 \mathrm{~ms}$ high and $17 \mathrm{~ms}$ _low conditions were stronger than those in the $17 \mathrm{~ms}$ _guess condition $(|t|>3.66, p<.002)$. Similar to the results of N170 amplitudes, stronger face-minus-house 
N170 component was also associated with higher subjective confidence, supporting the notion that N170 is a graded response.

The analyses conducted so far confirm that both the overall N170 and the face-minushouse N170 component grade with stimulus duration and subjective confidence that the stimulus was a face. However, a conclusion that the N170 is generated in a graded fashion would be premature. In the first half of the experiment, we presented all stimuli for $17 \mathrm{~ms}$, while in the second half the stimulus duration was either $17 \mathrm{~ms}$ or $200 \mathrm{~ms}$. It remains possible that, although the N170 is generated all-or-none, this discrepancy in parameters may result in distinct amplitudes of the "full" N170 in the two halves, which further led to the observed differences of N170 between $17 \mathrm{~ms}$ and $200 \mathrm{~ms}$ conditions in the above results. In other words, if the "full" N170 differed between the two halves, the conclusion that the N170 is generated in a graded manner would still be premature. In this case, the generation of the N170 needs to be tested with faces presented for different durations in the same blocks where the "full" N170 remains constant. On the contrary, if the "full" N170 is similar for the two halves, it would be safer to draw the conclusion that the N170 is graded. Therefore, it is critical to examine whether the "full" N170 was different between the first and second halves before drawing concrete conclusions on the N170 generation. To do so, we compared N170 amplitudes for faces that were presented for $17 \mathrm{~ms}$ and perceived with high subjective confidence in the two halves to explore whether their "full" N170 is comparable. We additionally inspected the amplitudes for faces presented for $17 \mathrm{~ms}$ and $200 \mathrm{~ms}$ with high subjective confidence in the same half to examine whether the N170 is generated in graded fashion or is all-or-none in nature. 


\subsection{Potentials from trials with high confidence}

The main point of interest in this study was to test the amplitude differences between durations when participants had similarly high subjective confidence about the nature of the stimulus. In the following analyses we only include trials in which intact stimuli were presented and high confidence was reported. Because we only included the $200 \mathrm{~ms}$ duration in the second half of the experiment, we created a new factor for the LMM analysis, Duration Half, which had three levels: 17ms_half1, 17ms_half2, and 200ms_half2.

The grand average ERP waveforms and topographic maps corresponding to the P1 and N170 time-windows are shown in Figure 6D and 6C, respectively. We submitted the LMM results to pairwise contrasts and pairwise interaction contrasts to test the influence of Duration Half on overall ERP amplitudes and the face-minus-house components of both P1 and N170.

\subsubsection{P1 amplitudes}

The estimated marginal means of P1 amplitudes in each condition are displayed in Figure 6A. Pairwise contrasts, corrected with Bonferroni methods for three tests, explored the influence of Duration Half on P1 amplitude. These contrasts reveal that all comparisons were equivalent to the null effect for both hemisphere electrodes $(|t|>2.49, p<.007)$. Pairwise interaction contrasts for the face-minus-house P1 component, again corrected with Bonferroni tests for three tests, revealed inconclusive results (NHST: $|t|<0.76, p>.99$; ET: $|t|<1.71, p>.13$ ) with the exception that the comparison between 17ms_half1 and $200 \mathrm{~ms} \_$half2 was equivalent to the null effect $(t(4863.0)=2.30, p=.03, b=-0.00,90 \% C I=$ $[-0.46,0.46])$. Overall, these results revealed no systematic influence of Half or Duration on P1 amplitudes for intact stimuli perceived with high confidence. 




Figure 6. ERP results for intact stimuli with high subjective confidence as a function of Hemisphere (left vs. right), Category (faces vs. houses), and Duration Half (17ms_half1, 17ms_half2, vs. 200ms_half2. $A$ and $B$ depict the estimated marginal means of P1 and N170 amplitudes, respectively; error bars represent 95\% confidence intervals. $C$, Topographic maps corresponding to the P1 and N170 components. The grey dots in the schematic brain denote the channel locations for both P1 and N170 on both hemispheres. $D$, The grand average ERP waveforms. The grey dashed rectangular denotes the time-window for the P1 $(96 \mathrm{~ms}-136 \mathrm{~ms})$, while the grey solid rectangular denotes the time windows for the N170 (152-192ms).

Please see Figure S3 in supplementary materials for colour topographic maps. 


\subsubsection{N170 amplitudes}

The estimated marginal means of N170 amplitudes in each condition are displayed in Figure 6B. Differences of N170 amplitudes between levels of Duration Half were compared with pairwise contrasts, corrected using Bonferroni corrections for three tests. These contrasts revealed two important results, which were similar for both hemispheres. First, N170 amplitudes were weaker for the $17 \mathrm{~ms}$ _half1 condition compared to those for both the $17 \mathrm{~ms} \_$half2 $($left: $t(18306.7)=3.97, p<.001, b=0.60,95 \% C I=[0.24,0.96]$; right: $t(18304.0)=6.59, p<.001, b=1.00,95 \% C I=[0.64,1.36])$ and 200ms_half2 (left: $t(17686.7)=4.38, p<.001, b=0.56,95 \% C I=[0.25,0.86] ;$ right: $t(17334.1)=7.17, p<$ $.001, b=0.91,95 \% C I=[0.61,1.22])$ conditions. These results, especially the differences between 17ms_half1 and 17ms_half2, suggest that the "full" N170 differs between the two halves of the experiment. Thus, it is critical to inspect the N170 generation with amplitudes in the same blocks, as shown following. In the second half of the experiment (i.e., the same blocks), the differences in N170 amplitudes between $17 \mathrm{~ms}$ and $200 \mathrm{~ms}$ conditions were statistically equivalent to the null effect (left: $t(18181.2)=2.79, p=.008, b=-0.04,90 \% C I$ $=[-0.39,0.30]$; right: $t(18079.2)=2.53, p=.02, b=-0.09,90 \% C I=[-0.43,0.26])$. This result suggests that a "full" N170 is generated once a face is perceived with high subjective confidence regardless of stimulus duration.

We also analysed the influence of Duration Half on the face-minus-house N170 component, again using pairwise interaction contrasts corrected with Bonferroni methods for three tests. We again found similar effects for both hemispheres. The differences in faceminus-house N170 component between 17ms_half1 and 17ms_half2 conditions were inconclusive for both hemispheres (NHST: $|t|<1.3, p>.68$; ET: $|t|<1.56, p>.17$ ). Nevertheless, the amplitudes in both conditions were weaker than those for the 200ms_half2 
condition $\left(17 \mathrm{~ms} \_\right.$half1 left hemisphere: $t(7112.0)=6.58, p<.001, b=1.56,95 \% C I=[0.99$, 2.12]; 17ms_half1 right hemisphere: $t(2326.2)=8.58, p<.001, b=1.97,95 \% C I=[1.42$, 2.52]; 17ms_half2 left: $t(13731.6)=6.68, p<.001, b=1.90,95 \% C I=[1.22,2.58]$; $17 \mathrm{~ms}$ half2 right: $t(7615.6)=6.82, p<.001, b=1.92,95 \% C I=[1.24,2.59])$. The faceminus-house N170 component was stronger when faces were presented for 200ms relative to $17 \mathrm{~ms}$, although stimuli were perceived with high subjective confidence in each case.

\section{Discussion}

We sought a resolution to the question of whether visual categorization is supported by graded or all-or-none neural mechanisms by inspecting the generation of the N170. To achieve this, we explored the influence of stimulus category (face vs. house; intact vs. scrambled) and duration (17ms vs. $200 \mathrm{~ms})$ on the N170 amplitudes, as well as their associations with subjective confidence. We analysed these components in three different ways. In our first analysis, in which we averaged across subjective confidence, we replicated previous observations (Carbon et al., 2005; Tanskanen et al., 2007) that N170 amplitude evoked by intact faces is greater for longer compared to shorter stimulus durations. This finding also concurs with earlier observations of the effects of stimulus onset asynchrony (Eiserbeck et al., 2021; Nasr, 2010) and visual noise (Bankó et al., 2011a; Jemel et al., 2003; Navajas et al., 2013; Philiastides et al., 2006; Philiastides \& Sajda, 2006; Schneider et al., 2007) on N170 amplitudes. These studies, and the results of our first analysis, suggest that stimuli with more facial information (temporal or spatial) elicit stronger N170 (or M170) responses. In other words, without considering subjective confidence, our first analysis appears to support the hypothesis that the N170 is a graded response. However, neither our first analysis nor those presented in the earlier studies can rule out the possibility that the 
N170 amplitudes observed for brief stimuli are lower because the response is diluted by including trials in which the face stimulus was not detected.

Since detected faces usually evoke greater N170 responses than undetected faces (Fisch et al., 2009; Navajas et al., 2013; Rodríguez et al., 2012), our second analysis explored N170 amplitudes further by considering subjective confidence. In the critical conditions, in which stimuli were correctly categorized with equally high subjective confidence, faces presented for longer durations still evoked greater N170 amplitudes. This finding also appears to support the hypothesis that N170 is a graded response that reflects the subjective strength of the percept of a face. However, this conclusion, too, was undermined because the discrepancy in parameters may lead to differences in the "full" N170 between the first and second halves of the experiment, which in turn could explain the different N170 amplitudes for varying durations. Accordingly, we further examined differences between the N170 evoked by briefly presented faces in the two halves, as well as between faces presented for $17 \mathrm{~ms}$ or $200 \mathrm{~ms}$ in the second half of the recording session.

Our final analysis first revealed that N170 amplitudes evoked by faces presented for $17 \mathrm{~ms}$ and perceived with high subjective confidence were stronger in the second half of the experiment than the first. This might stem from lack of previous knowledge of the faces during the first half where all stimuli were presented for $17 \mathrm{~ms}$. Participants may not have had a clear idea of what the face images looked like until they saw the longer-duration stimuli in the second half. In spite of the fact that N170 amplitudes evoked by intact faces for $17 \mathrm{~ms}$ were stronger in the second half of the experiment, the face-minus-house component was more likely to remain comparable between the two halves. Hence, the differences of the N170 between halves should be attributable to the non-face contribution to the N1/N170 complex. This implies that no matter what causes the stronger N170 in the second halves, the influence on the N170 is not specific to faces. 
Since the "full" N170 differs between the first and second halves of the experiment, the N170 generation should be tested within the same blocks. In comparing N170 amplitudes from the same half, the conclusion that the N170 is a graded response is undermined. Specifically, ET revealed that N170 amplitudes in the second half were comparable between $17 \mathrm{~ms}$ and $200 \mathrm{~ms}$ conditions when faces were perceived with high subjective confidence. This analysis suggests that rather than being a graded response, the N170 is generated in an all-ornone manner once a face is perceived. All stimuli that were correctly perceived as faces with high subjective confidence appear to have evoked similar N170 amplitudes, regardless of stimulus duration. This conclusion implies that the appearance that N170 grades in strength with stimulus information or subjective confidence is an artefact of mixing heterogeneous trial types in signal averaging.

Distinct from the comparable N170 amplitudes in the $17 \mathrm{~ms}$ and $200 \mathrm{~ms}$ conditions with high subjective confidence, the face-minus-house N170 component was higher in amplitude in the $200 \mathrm{~ms}$ condition than in the $17 \mathrm{~ms}$ condition when similar high subjective confidence was reported. This result does not seem to be consistent with a recent study which employed rapid serial visual presentation and did not observe the differences in face-minusnonface signals evoked by stimuli presented for different durations (Retter et al., 2020). This inconsistency may result from the different methods used in the two studies and, therefore, the signals measured may reflect different aspects of ERPs. More specifically, Retter and colleagues (2020) analysed the implicit frequency-tagged face-minus-nonface EEG responses, which are likely to reflect the combined contributions of both early and late ERP components. In other words, their results may suggest that the mixed combinations of faceminus-nonface P1, N170, N250 and other later components were comparable when faces were perceived regardless of stimulus duration. By contrast, our study estimated the ERPs for each component directly. These results suggest that the face-minus-house N170 component 
was stronger for stimuli presented for longer durations. This finding, in addition to the observation of comparable N170 for faces with varying duration, indicates that although stimulus duration impacted the general component, potentially also the face-minus-house N1/N170 component, a "full" N170 is always achieved whenever a face is perceived, corroborating that the N170 is generated in all-or-none.

In addition to the N170, we also examined P1 amplitudes in each condition. We found similar P1 amplitudes evoked by intact stimuli in $17 \mathrm{~ms}$ and $200 \mathrm{~ms}$ conditions. However, some previous studies have reported that faces embedded in noise evoked stronger P1 amplitudes (Bankó et al., 2011; Rousselet et al., 2008; Schneider et al., 2007; Tanskanen et al., 2005; Tarkiainen et al., 2002; but see Jemel et al., 2003). The discrepancy might result from the fact that embedding faces in noise alters the low-level properties of stimuli, as well as the facial information. In contrast, the low-level information of stimuli remains the same when stimuli were presented for various durations, further leading to comparable P1 amplitudes.

The only factor affecting the P1 amplitudes observed in this study was subjective confidence. Stronger P1 amplitudes were associated with higher confidence for categorization, and the strongest P1 amplitudes were observed when participants were sure what the stimuli were. Interestingly, the N170 for faces was also affected by the subjective confidence in the similar way, i.e., stronger N170 amplitudes being linked with higher confidence. These connections may indicate that the perception of low-level information (indexed by the stronger P1) is a prerequisite for the possible higher-level processing, e.g., categorization with high confidence (indexed by the stronger N170). Specifically, stronger P1 may reflect more attention was allocated on the stimuli, which renders it possible to perceive the stimuli with high subjective confidence. 
In this study, we grouped N170 amplitudes by participants' subjective confidence, which should reflect their subjective perception, and observed that the effects of durations on the N170 disappeared, suggesting that weaker N170 reflects the uncertainty of face perception but not the direct impact of limited stimulus durations. Since faces with shorter durations or more noise are both associated with lower stimulus visibility, it may be that similar differences in subjective perception, rather than the direct effect of noise, are linked with N170 differences in previous studies (Bestelmeyer et al., 2008; Horovitz et al., 2004; Németh et al., 2014; Tarkiainen et al., 2002). It is not a coincidence that subjective perception can dissociate trials generating full N170 from others, as the N170 is believed to be the index of subjective face perception/awareness (Rossion, 2014; Rossion \& Jacques, 2011). These results provide further support to categorisation of faces occurring in all-or-none fashion.

Given the relationships between subjective perception and the N170(/P1), we may need to re-consider the conclusions of other neural indicators drawn from previous studies where subjective perception might vary in different experimental conditions. For example, apart from the N170, stimulus visibility, which was controlled by shortening stimulus durations or adding noise, was also found to modulate the N1 (Bacon-Macé et al., 2005; Haider et al., 1964), N250 (Carbon et al., 2005; Jemel et al., 2010), P300/M300 (Carbon et al., 2005; Philiastides \& Sajda, 2006; Tanskanen et al., 2007), other ERP components (Jemel et al., 2010; Nasr, 2010), and even neuro-imaging responses (Bar et al., 2001; Natu et al., 2016). For these studies, caution is needed in drawing conclusions of a graded response before taking into account subjective perception of the stimuli.

We applied some relatively new statistical methods in this study. First, LMM incorporating both by-subject and by-stimulus random effects enables us to generalize our results not only to similar participants, but also to similar stimuli. Moreover, in addition to the conventional NHST, which can test whether there are significant differences but provides no 
mechanism for endorsing the null hypothesis (Cohen, 1994), we employed ET to test whether the effects of interest are compatible with the null hypothesis. ET is of great importance in providing evidence for accepting amplitude differences between durations being equivalent to null effects, which corroborates the all-or-none hypothesis.

In summary, our study showed that the "full" N170 is generated once a face is perceived with high subjective confidence, supporting the all-or-none hypothesis for the N170 generation. These results further suggest that categorisation of faces also occurs in a similar all-or-none fashion. 


\section{Contributions:}

Haiyang Jin: conceptualization, data curation, formal analysis, validation, investigation, methodology, project administration, resources, visualization, writing original draft, writing - review \& editing

William G. Hayward: conceptualization, funding acquisition, supervision, writing review \& editing

Paul M. Corballis: conceptualization, funding acquisition, supervision, writing review \& editing

\section{Funding:}

This work was supported by a China Scholarship Council grant ([2015]08330295) awarded to Haiyang Jin and co-funded by a grant (HKU17608519) from the General Research Fund of the Hong Kong Research Grants Council to William G. Hayward.

\section{Acknowledgment:}

The authors thank Dr. Veema Lodhia for assistance with data collection and wish to acknowledge the use of New Zealand eScience Infrastructure (NeSI) high performance computing facilities, consulting support and/or training services as part of this research. New Zealand's national facilities are provided by NeSI and funded jointly by NeSI's collaborator institutions and through the Ministry of Business, Innovation \& Employment's Research Infrastructure programme. URL https://www.nesi.org.nz. Conflict of interest: The authors declare no competing interests. 


\section{Reference}

Aarts, E., Verhage, M., Veenvliet, J. V., Dolan, C. V., \& Van Der Sluis, S. (2014). A solution to dependency: Using multilevel analysis to accommodate nested data. Nature Neuroscience, 17(4), 491-496. https://doi.org/10.1038/nn.3648

Acunzo, D. J., MacKenzie, G., \& van Rossum, M. C. W. (2012). Systematic biases in early ERP and ERF components as a result of high-pass filtering. Journal of Neuroscience Methods, 209(1), 212-218. https://doi.org/10.1016/j.jneumeth.2012.06.011

Akaike, H. (1998). Information Theory and an Extension of the Maximum Likelihood Principle. In Selected Papers of Hirotugu Akaike (pp. 199-213). https://doi.org/10.1007/978-1-4612-1694-0_15

Baayen, R. H., Tweedie, F. J., \& Schreuder, R. (2002). The Subjects as a Simple Random Effect Fallacy: Subject Variability and Morphological Family Effects in the Mental Lexicon. Brain and Language, 81(1-3), 55-65. https://doi.org/10.1006/brln.2001.2506

Bacon-Macé, N., Macé, M. J. M., Fabre-Thorpe, M., \& Thorpe, S. J. (2005). The time course of visual processing: Backward masking and natural scene categorisation. Vision Research, 45(11), 1459-1469. https://doi.org/10.1016/j.visres.2005.01.004

Bankó, E. M., Gál, V., Körtvélyes, J., Kovács, G., \& Vidnyánszky, Z. (2011a). Dissociating the effect of noise on sensory processing and overall decision difficulty. Journal of Neuroscience, 31(7), 2663-2674. https://doi.org/10.1523/JNEUROSCI.2725-10.2011

Bar, M., Tootell, R. B. H., Schacter, D. L., Greve, D. N., Fischl, B., Mendola, J. D., Rosen, B. R., \& Dale, A. M. (2001). Cortical Mechanisms Specific to Explicit Visual Object Recognition. Neuron, 29(2), 529-535. https://doi.org/10.1016/S0896-6273(01)002240

Bates, D., Kliegl, R., Vasishth, S., \& Baayen, R. H. (2015). Parsimonious Mixed Models. 
Bates, D., Mächler, M., Bolker, B. M., \& Walker, S. (2015). Fitting Linear Mixed-Effects Models Using lme4. Journal of Statistical Software, 67(1), 361-373. https://doi.org/10.18637/jss.v067.i01

Bentin, S., Allison, T., Puce, A., Perez, E., \& McCarthy, G. (1996). Electrophysiological Studies of Face Perception in Humans. Journal of Cognitive Neuroscience, 8(6), 551565. https://doi.org/10.1162/jocn.1996.8.6.551

Bestelmeyer, P. E. G., Jones, B. C., DeBruine, L. M., Little, A. C., Perrett, D. I., Schneider, A., Welling, L. L. M., \& Conway, C. A. (2008). Sex-contingent face aftereffects depend on perceptual category rather than structural encoding. Cognition, 107(1), 353-365. https://doi.org/10.1016/j.cognition.2007.07.018

Boisgontier, M. P., \& Cheval, B. (2016). The anova to mixed model transition. Neuroscience \& Biobehavioral Reviews, 68, 1004-1005. https://doi.org/10.1016/j.neubiorev.2016.05.034

Brauer, M., \& Curtin, J. J. (2018). Linear mixed-effects models and the analysis of nonindependent data: A unified framework to analyze categorical and continuous independent variables that vary within-subjects and/or within-items. Psychological Methods, 23(3), 389-411. https://doi.org/10.1037/met0000159

Carbon, C.-C., Schweinberger, S. R., Kaufmann, J. M., \& Leder, H. (2005). The Thatcher illusion seen by the brain: An event-related brain potentials study. Cognitive Brain Research, 24(3), 544-555. https://doi.org/10.1016/j.cogbrainres.2005.03.008

Chaumon, M., Bishop, D. V. M., \& Busch, N. A. (2015). A practical guide to the selection of independent components of the electroencephalogram for artifact correction. Journal of Neuroscience Methods, 250, 47-63. https://doi.org/10.1016/j.jneumeth.2015.02.025

Cohen, J. (1994). The earth is round ( $<$.05). American Psychologist, 49(12), 997-1003. https://doi.org/10.1037/0003-066X.49.12.997 
Delorme, A., \& Makeig, S. (2004). EEGLAB: An open source toolbox for analysis of singletrial EEG dynamics including independent component analysis. Journal of Neuroscience Methods, 134(1), 9-21. https://doi.org/10.1016/j.jneumeth.2003.10.009

Eiserbeck, A., Enge, A., Rabovsky, M., \& Abdel Rahman, R. (2021). Electrophysiological Chronometry of Graded Consciousness during the Attentional Blink. Cerebral Cortex, bhab289. https://doi.org/10.1093/cercor/bhab289

Ferree, T. C., Luu, P., Russell, G. S., \& Tucker, D. M. (2001). Scalp electrode impedance, infection risk, and EEG data quality. Clinical Neurophysiology, 112(3), 536-544. https://doi.org/10.1016/S1388-2457(00)00533-2

Fisch, L., Privman, E., Ramot, M., Harel, M., Nir, Y., Kipervasser, S., Andelman, F., Neufeld, M. Y., Kramer, U., Fried, I., \& Malach, R. (2009). Neural "Ignition": Enhanced Activation Linked to Perceptual Awareness in Human Ventral Stream Visual Cortex. Neuron, 64(4), 562-574. https://doi.org/10.1016/j.neuron.2009.11.001

Ge, L., Zhang, H., Wang, Z., Quinn, P. C., Pascalis, O., Kelly, D. J., Slater, A., Tian, J., \& Lee, K. (2009). Two faces of the other-race effect: Recognition and categorisation of Caucasian and Chinese faces. Perception, 38(8), 1199-1210. https://doi.org/10.1068/p6136

Haider, M., Spong, P., \& Lindsley, D. B. (1964). Attention, vigilance, and cortical evokedpotentials in humans. Science, 145(3628), 180-182. https://doi.org/10.1126/science.145.3628.180

Helle, C., \& Perona. (2000). Caltech Houses Database. http://www.vision.caltech.edu/htmlfiles/archive.html

Horovitz, S. G., Rossion, B., Skudlarski, P., \& Gore, J. C. (2004). Parametric design and correlational analyses help integrating fMRI and electrophysiological data during face 
processing. NeuroImage, 22(4), 1587-1595.

https://doi.org/10.1016/j.neuroimage.2004.04.018

Jaeger, T. F. (2008). Categorical data analysis: Away from ANOVAs (transformation or not) and towards logit mixed models. Journal of Memory and Language, 59(4), 434-446. https://doi.org/10.1016/j.jml.2007.11.007

Jemel, B., Schuller, A.-M., Cheref-Khan, Y., Goffaux, V., Crommelinck, M., \& Bruyer, R. (2003). Stepwise emergence of the face-sensitive N170 event-related potential component. Neuroreport, 14(16), 2035-2039. https://doi.org/10.1097/00001756200311140-00006

Jemel, B., Schuller, A.-M., \& Goffaux, V. (2010). Characterizing the Spatio-temporal Dynamics of the Neural Events Occurring prior to and up to Overt Recognition of Famous Faces. Journal of Cognitive Neuroscience, 22(10), 2289-2305. https://doi.org/10.1162/jocn.2009.21320

Jin, H. (2021). Hello again, ANOVA: Rethinking ANOVA in the context of confirmatory data analysis [Preprint]. PsyArXiv. https://doi.org/10.31234/osf.io/yhmzg

Kliegl, R., Wei, P., Dambacher, M., Yan, M., \& Zhou, X. (2011). Experimental effects and individual differences in linear mixed models: Estimating the relationship between spatial, object, and attraction effects in visual attention. Frontiers in Psychology, I(JAN), 1-12. https://doi.org/10.3389/fpsyg.2010.00238

Kristensen, M., \& Hansen, T. (2004). Statistical analyses of repeated measures in physiological research: A tutorial. Advances in Physiology Education, 28(1), 2-14. https://doi.org/10.1152/advan.00042.2003

Kuefner, D., Jacques, C., Prieto, E. A., \& Rossion, B. (2010). Electrophysiological correlates of the composite face illusion: Disentangling perceptual and decisional components of 
holistic face processing in the human brain. Brain and Cognition, 74(3), 225-238. https://doi.org/10.1016/j.bandc.2010.08.001

Lakens, D. (2017). Equivalence Tests: A Practical Primer for t Tests, Correlations, and MetaAnalyses. Social Psychological and Personality Science, 8(4), 355-362. https://doi.org/10.1177/1948550617697177

Lakens, D., Scheel, A. M., \& Isager, P. M. (2018). Equivalence Testing for Psychological Research: A Tutorial. Advances in Methods and Practices in Psychological Science, 1(2), 259-269. https://doi.org/10.1177/2515245918770963

Lenth, R. (2019). emmeans: Estimated Marginal Means, aka Least-Squares Means.

Linck, J. A., \& Cunnings, I. (2015). The Utility and Application of Mixed-Effects Models in Second Language Research. Language Learning, 65(S1), 185-207. https://doi.org/10.1111/lang.12117

Magezi, D. A. (2015). Linear mixed-effects models for within-participant psychology experiments: An introductory tutorial and free, graphical user interface (LMMgui). Frontiers in Psychology, 6. https://doi.org/10.3389/fpsyg.2015.00002

Mara, C. A., \& Cribbie, R. A. (2012). Paired-Samples Tests of Equivalence. Communications in Statistics - Simulation and Computation, 41(10), 1928-1943. https://doi.org/10.1080/03610918.2011.626545

Matuschek, H., Kliegl, R., Vasishth, S., Baayen, R. H., \& Bates, D. (2017). Balancing Type I error and power in linear mixed models. Journal of Memory and Language, 94, 305315. https://doi.org/10.1016/j.jml.2017.01.001

Meyners, M. (2012). Equivalence tests - A review. Food Quality and Preference, 26(2), 231-245. https://doi.org/10.1016/j.foodqual.2012.05.003 
Mitsudo, T., Kamio, Y., Goto, Y., Nakashima, T., \& Tobimatsu, S. (2011). Neural responses in the occipital cortex to unrecognizable faces. Clinical Neurophysiology, 122(4), 708-718. https://doi.org/10.1016/j.clinph.2010.10.004

Miyakoshi, M. (2019). Makoto's preprocessing pipeline. Swartz Center for Computational Neuroscience. https://sccn.ucsd.edu/wiki/Makoto's_preprocessing_pipeline

Mognon, A., Jovicich, J., Bruzzone, L., \& Buiatti, M. (2011). ADJUST: An automatic EEG artifact detector based on the joint use of spatial and temporal features. Psychophysiology, 48(2), 229-240. https://doi.org/10.1111/j.1469-8986.2010.01061.x

Nasr, S. (2010). Differential impact of attention on the early and late categorization related human brain potentials. Journal of Vision, 10(11), 18-18. https://doi.org/10.1167/10.11.18

Natu, V. S., Barnett, M. A., Hartley, J., Gomez, J., Stigliani, A., \& Grill-Spector, K. (2016). Development of Neural Sensitivity to Face Identity Correlates with Perceptual Discriminability. The Journal of Neuroscience, 36(42), 10893-10907. https://doi.org/10.1523/JNEUROSCI.1886-16.2016

Navajas, J., Ahmadi, M., \& Quian Quiroga, R. (2013). Uncovering the Mechanisms of Conscious Face Perception: A Single-Trial Study of the N170 Responses. Journal of Neuroscience, 33(4), 1337-1343. https://doi.org/10.1523/JNEUROSCI.1226-12.2013

Németh, K., Kovács, P., Vakli, P., Kovács, G., \& Zimmer, M. (2014). Phase noise reveals early category-specific modulation of the event-related potentials. Frontiers in Psychology, 5(APR), 367. https://doi.org/10.3389/fpsyg.2014.00367

Nemrodov, D., Niemeier, M., Mok, J. N. Y., \& Nestor, A. (2016). The time course of individual face recognition: A pattern analysis of ERP signals. NeuroImage, 132, 469-476. https://doi.org/10.1016/j.neuroimage.2016.03.006 
Nolan, H., Whelan, R., \& Reilly, R. B. (2010). FASTER: Fully Automated Statistical Thresholding for EEG artifact Rejection. Journal of Neuroscience Methods, 192(1), 152-162. https://doi.org/10.1016/j.jneumeth.2010.07.015

Philiastides, M. G., Ratcliff, R., \& Sajda, P. (2006). Neural Representation of Task Difficulty and Decision Making during Perceptual Categorization: A Timing Diagram. Journal of Neuroscience, 26(35), 8965-8975. https://doi.org/10.1523/JNEUROSCI.165506.2006

Philiastides, M. G., \& Sajda, P. (2006). Temporal Characterization of the Neural Correlates of Perceptual Decision Making in the Human Brain. Cerebral Cortex, 16(4), 509518. https://doi.org/10.1093/cercor/bhi130

Piazza, C., Miyakoshi, M., Akalin-Acar, Z., Cantiani, C., Reni, G., Bianchi, A. M., \& Makeig, S. (2016). An Automated Function for Identifying EEG Independent Components Representing Bilateral Source Activity. In E. Kyriacou, S. Christofides, \& C. S. Pattichis (Eds.), XIV Mediterranean Conference on Medical and Biological Engineering and Computing 2016 (pp. 105-109). Springer International Publishing. https://doi.org/10.1007/978-3-319-32703-7_22

Pion-Tonachini, L., Kreutz-Delgado, K., \& Makeig, S. (2019). ICLabel: An automated electroencephalographic independent component classifier, dataset, and website. NeuroImage, 198, 181-197. https://doi.org/10.1016/j.neuroimage.2019.05.026

Quené, H., \& van den Bergh, H. (2004). On multi-level modeling of data from repeated measures designs: A tutorial. Speech Communication, 43(1-2), 103-121. https://doi.org/10.1016/j.specom.2004.02.004

R Core Team. (2018). R: A language and environment for statistical computing. $\mathrm{R}$ Foundation for Statistical Computing. 
Retter, T. L., Jiang, F., Webster, M. A., \& Rossion, B. (2020). All-or-none face categorization in the human brain. NeuroImage, 213, 116685. https://doi.org/10.1016/j.neuroimage.2020.116685

Rodríguez, V., Thompson, R., Stokes, M., Brett, M., Alvarez, I., Valdes-Sosa, M., \& Duncan, J. (2012). Absence of Face-specific Cortical Activity in the Complete Absence of Awareness: Converging Evidence from Functional Magnetic Resonance Imaging and Event-related Potentials. Journal of Cognitive Neuroscience, 24(2), 396-415. https://doi.org/10.1162/jocn_a_00137

Rogers, J. L., Howard, K. I., \& Vessey, J. T. (1993). Using significance tests to evaluate equivalence between two experimental groups. Psychological Bulletin, 113(3), 553565. https://doi.org/10.1037/0033-2909.113.3.553

Rossion, B. (2014). Understanding face perception by means of human electrophysiology. Trends in Cognitive Sciences, 18(6), 310-318. https://doi.org/10.1016/j.tics.2014.02.013

Rossion, B., \& Caharel, S. (2011). ERP evidence for the speed of face categorization in the human brain: Disentangling the contribution of low-level visual cues from face perception. Vision Research, 51(12), 1297-1311. https://doi.org/10.1016/j.visres.2011.04.003

Rossion, B., \& Jacques, C. (2008). Does physical interstimulus variance account for early electrophysiological face sensitive responses in the human brain? Ten lessons on the N170. NeuroImage, 39(4), 1959-1979. https://doi.org/10.1016/j.neuroimage.2007.10.011

Rossion, B., \& Jacques, C. (2011). The N170: Understanding the Time Course of Face Perception in the Human Brain. In The Oxford Handbook of Event-Related Potential 
Components (pp. 1-30). Oxford University Press.

https://doi.org/10.1093/oxfordhb/9780195374148.013.0064

Rousselet, G. A., Pernet, C. R., Bennett, P. J., \& Sekuler, A. B. (2008). Parametric study of EEG sensitivity to phase noise during face processing. BMC Neuroscience, 9(1), 98. https://doi.org/10.1186/1471-2202-9-98

RStudio Team. (2019). RStudio: Integrated Development for R. RStudio. RStudio, Inc.

Schneider, B. L., DeLong, J. E., \& Busey, T. A. (2007). Added noise affects the neural correlates of upright and inverted faces differently. Journal of Vision, 7(4), 4-4. https://doi.org/10.1167/7.4.4

Schweinberger, S. R., \& Neumann, M. F. (2016). Repetition effects in human ERPs to faces. Cortex, 80, 141-153. https://doi.org/10.1016/j.cortex.2015.11.001

Seaman, M. A., \& Serlin, R. C. (1998). Equivalence confidence intervals for two-group comparisons of means. Psychological Methods, 3(4), 403-411. https://doi.org/10.1037/1082-989X.3.4.403

Smith, M. L. (2012). Rapid processing of emotional expressions without conscious awareness. Cerebral Cortex, 22(8), 1748-1760. https://doi.org/10.1093/cercor/bhr250

Tanskanen, T., Näsänen, R., Montez, T., Päällysaho, J., \& Hari, R. (2005). Face Recognition and Cortical Responses Show Similar Sensitivity to Noise Spatial Frequency. Cerebral Cortex, 15(5), 526-534. https://doi.org/10.1093/cercor/bhh152

Tanskanen, T., Näsänen, R., Ojanpää, H., \& Hari, R. (2007). Face recognition and cortical responses: Effect of stimulus duration. NeuroImage, 35(4), 1636-1644. https://doi.org/10.1016/j.neuroimage.2007.01.023

Tarkiainen, A., Cornelissen, P. L., \& Salmelin, R. (2002). Dynamics of visual feature analysis and object-level processing in face versus letter-string perception. Brain, 125(5), 1125-1136. https://doi.org/10.1093/brain/awf112 
Tucker, D. M. (1993). Spatial sampling of head electrical fields: The geodesic sensor net. Electroencephalography and Clinical Neurophysiology, 87(3), 154-163. https://doi.org/10.1016/0013-4694(93)90121-B

Weber, R., \& Popova, L. (2012). Testing Equivalence in Communication Research: Theory and Application. Communication Methods and Measures, 6(3), 190-213. https://doi.org/10.1080/19312458.2012.703834

Wickham, H. (2017). tidyverse: Easily Install and Load the "Tidyverse" (R package version $1.2 .1)$

Willenbockel, V., Sadr, J., Fiset, D., Horne, G. O., Gosselin, F., \& Tanaka, J. W. (2010). Controlling low-level image properties: The SHINE toolbox. Behavior Research Methods, 42(3), 671-684. https://doi.org/10.3758/BRM.42.3.671

Winkler, I., Debener, S., Muller, K. R., \& Tangermann, M. (2015). On the influence of highpass filtering on ICA-based artifact reduction in EEG-ERP. Proceedings of the Annual International Conference of the IEEE Engineering in Medicine and Biology Society, EMBS, 2015-Novem, 4101-4105.

https://doi.org/10.1109/EMBC.2015.7319296 